\title{
ALOCAÇÃO DE BOLSAS DE INICIAÇÃO CIENTÍFICA ÀS UNIDADES DA EMBRAPA COM MODELOS DE ANÁLISE ENVOLTÓRIA DE DADOS COM GANHOS DE SOMA ZERO
}

\author{
Eliane Gonçalves Gomes ${ }^{1}$ \\ Geraldo da Silva e Souza ${ }^{1}$ \\ Suzana Maria Valle Lima ${ }^{2}$ \\ Carlos Eduardo Lazarini da Fonseca ${ }^{2}$
}

\begin{abstract}
Resumo: A alocação de recursos é um dos problemas de interesse para gestores de organizações, notadamente quando diante de sua escassez ou limitação. Este artigo tem como objetivo usar um modelo de produção baseado na Análise Envoltória de Dados (DEA) com Ganhos de Soma Zero (DEA-GSZ), para alocar bolsas de iniciação científica aos centros de pesquisa da Empresa Brasileira de Pesquisa Agropecuária (Embrapa). As unidades sob avaliação são os 37 centros de pesquisa da empresa. Como variável produto usou-se a alocação de cotas bolsas, e os insumos são proxies para produção científica, complexidade dos projetos de pesquisa, importância da participação de bolsistas nos projetos de pesquisa e o potencial para supervisão dos bolsistas.
\end{abstract}

Palavras-chave: Alocação de recursos; Eficiência; DEA; Ganhos de Soma Zero.

Abstract: Resource allocation is of concern to managers, specially when facing scarce or restricted resources. In this paper we use Zero Sum Gains Data Envelopment Analysis models (ZSG-DEA) to allocate scientific scholarships to the Brazilian Agricultural Research Corporation (Embrapa) research centers. The decision making units are the corporation's 37 research centers. The output measurement in the production process is the number of scholarships, and the input variables are quantitative proxies for scientific production, complexity of research projects, importance of scholarships for research projects and the supervision capability.

Key-words: Resource allocation; Efficiency; DEA; Zero Sum Gains.

\section{INTRODUÇÃO}

A Empresa Brasileira de Pesquisa Agropecuária (Embrapa) atua por intermédio de 37 Centros de Pesquisa, localizados em quase todos os estados brasileiros, nas mais diferentes condições ecológicas. Nesses centros, alunos de diferentes níveis de educacionais são admitidos para realização de estágios. Uma das formas de estágio para alunos que cursam o ensino superior é a iniciação científica, que tem como objetivo despertar e incentivar a vocação

\footnotetext{
1 Empresa Brasileira de Pesquisa Agropecuária (Embrapa) - Secretaria de Gestão e Estratégia, eliane.gomes@embrapa.br; geraldo.souza@embrapa.br

${ }^{2}$ Empresa Brasileira de Pesquisa Agropecuária (Embrapa) - Departamento de Pesquisa e Desenvolvimento, suzana.lima@embrapa.br; carlos.lazarini@embrapa.br
} 
científica entre estudantes de graduação e, assim, contribuir para a formação de recursos humanos para a pesquisa. A remuneração dessa forma de estágio é por meio de bolsas de iniciação científica. Para essa modalidade, além de recursos próprios, a Embrapa recebe bolsas do PIBIC (Programa Institucional de Bolsas de Iniciação Científica), concedidas pelo CNPq (Conselho Nacional de Desenvolvimento Científico e Tecnológico), e aloca-as entre os seus centros de pesquisa. Quando do recebimento de novas cotas de bolsas, é formado um comitê interno à empresa, encarregado de eleger critérios que sirvam de base para essa alocação.

As premissas que servem de base para a seleção dos critérios de distribuição de cotas referem-se tanto às características da Embrapa, quanto aos objetivos do CNPq para a concessão. Destacam-se: as normas estabelecidas pelo $\mathrm{CNPq}$ para o julgamento e a distribuição de bolsas do PIBIC; a forma de organização do trabalho de pesquisa na Embrapa (por projetos de pesquisa, em que projetos de maior complexidade implicam maior necessidade de apoio técnico); o estímulo à interação entre os centros de pesquisa e as universidades de onde os bolsistas se originam; a necessidade de tornar mais eqüitativa a disponibilidade de apoio técnico nos diferentes centros de pesquisa da Embrapa.

Para que um centro de pesquisa seja elegível para o recebimento de cotas de bolsas do PIBIC, os supervisores, além de serem detentores do título de doutor, devem ser líderes de projetos nos chamados Macroprogramas da Embrapa (os Macroprogramas são figuras programáticas de nível tático do sistema de gestão da empresa, orientadas para o gerenciamento de carteiras de projetos e processos). Para a distribuição de cotas para o período 2005/2006, o comitê elegeu três critérios para a classificação das unidades, que tomaram como base os tipos de projeto dos Macroprogramas liderados pela unidade, o número de pesquisadores doutores e as cotas atuais de bolsas do PIBIC.
Considerar diversos critérios para realizar uma distribuição justa de recursos é um dos problemas clássicos abordados pela Pesquisa Operacional, em especial, pelos modelos de programação matemática. O uso de modelos de Análise Envoltória de Dados (Data Envelopment Analysis - DEA) (COOPER et al., 2000) nesse contexto tem sido objeto de estudo na literatura, conforme apresentado em Beasley (2001), Yan et al. (2002), Gomes (2003), Lins et al. (2003), Korhonen e Syrjänen (2004), Lozano, Villa (2004), Gomes et al., 2005).

Os modelos DEA medem a eficiência de unidades produtivas e essa medida serve, normalmente, para avaliar as unidades e indicar quais as melhores práticas gerenciais que servem de referência para as demais unidades. A medida de eficiência pode ser considerada como um índice de aproveitamento de recursos e, assim, pode ser usada para a alocação e realocação dos mesmos. Devem-se alocar mais recursos para as unidades que melhor fazem uso deles. Esse tipo de procedimento é de interesse especial quando há um controle central que deve alocar/realocar recursos escassos (ou restritos) às unidades constituintes.

São aqui usados modelos baseados em DEA para a alocação das bolsas de iniciação científica aos centros de pesquisa da Embrapa. São usados modelos DEA com Ganhos de Soma Zero (DEA-GSZ) (GOMES, 2003; LINS et al., 2003; GOMES et al., 2005; GOMES, LINS, 2007), que permitem a realocação de recursos, mantendo-se o total inalterado (soma dos recursos constante). Como os resultados do modelo são valores contínuos, foi feita uma aproximação para este caso discreto.

A abordagem aqui proposta é aplicada à distribuição de bolsas de iniciação científica do PIBIC aos 37 centros de pesquisa da Embrapa. O modelo DEA formulado considera como insumos proxies de produção científica, complexidade dos projetos de pesquisa, importância das bolsas e potencial para supervisão dos bolsistas, e como produto as cotas de bolsas de cada unidade. 


\section{ANÁLISE ENVOLTÓRIA DE DADOS}

Modelos DEA têm como objetivo medir a eficiência de unidades tomadoras de decisão, designadas por DMUs (Decision Making Units), na presença de múltiplos inputs (entradas, insumos, recursos ou fatores de produção) e múltiplos outputs (saídas ou produtos).

Há dois modelos DEA clássicos: CCR e BCC. O modelo CCR (também conhecido por CRS ou constant returns to scale), trabalha com retornos constantes de escala (CHARNES et al., 1978). Esse modelo maximiza o quociente entre a combinação linear dos produtos e a combinação linear dos insumos, com a restrição de que para qualquer DMU esse quociente não pode ser maior que 1 . O modelo BCC (BANKER et al., 1984), também chamado de VRS (variable returns to scale), considera situações de eficiência de produção com variação de escala e não assume proporcionalidade entre insumos e produtos.

Em (1) apresenta-se a formulação matemática do modelo DEA CCR, no qual considera-se que cada DMU $k, k=$ $1 . . . n$, é uma unidade de produção que utiliza $m$ insumos $x_{i k}, i=1 \ldots m$, para produzir $s$ produtos $y_{j k}, j=1 . . . s ; h_{o}$ é a eficiência da DMU o em análise; $x_{i o}$ e $y_{j o}$ são os insumos e produtos da DMU $o ; v_{\mathrm{i}}$ e $u_{\mathrm{j}}$ são os pesos calculados pelo modelo para insumos e produtos, respectivamente.

$\max h_{o}=\sum_{j=1}^{s} u_{j} y_{j o}$

sujeito a

$\sum_{i=1}^{m} v_{i} x_{i o}=1$

$\sum_{j=1}^{s} u_{j} y_{j k}-\sum_{i=1}^{m} v_{i} x_{i k} \leq 0, \quad \forall k$

$u_{j}, v_{i} \geq 0 \quad \forall i, j$

\subsection{Modelos DEA com Ganhos de Soma Zero (DEA-GSZ)}

Modelos DEA-GSZ (GOMES, 2003; Lins et al., 2003; Gomes et al., 2005) assumem a hipótese de dependência de produção ou de uso dos recursos. Isto significa que há limitação ou à quantidade total a ser produzida ou ao compartilhamento de recursos. Assim, é imposta uma restrição adicional aos modelos DEA clássicos, qual seja, a de que a soma de um certo recurso (ou produto) deve ser constante. Ou seja, quando uma DMU ineficiente busca seu alvo na fronteira de eficiência, o valor das demais DMUs é alterado para manter a soma constante.

Há a possibilidade de mais de uma DMU procurar maximizar a eficiência. No paradigma do DEA-GSZ, uma busca em cooperação significa que as DMUs deste grupo tentam retirar determinada quantidade de produto (ou dar insumo) apenas das DMUs não pertencentes ao grupo. No caso em que todas as DMUs ineficientes formam um único grupo de cooperação, a aplicação do modelo DEAGSZ fará com que ocorra a redistribuição do insumo ou do produto de soma constante. Após essa redistribuição, todas as DMUs pertencerão à fronteira de eficiência, ou seja, a eficiência média global será $100 \%$. Essa situação vai de encontro aos objetivos de uma administração central em relação aos recursos, qual seja, promover maior eficiência no uso dos mesmos e ter suas unidades constituintes operando com a máxima eficiência.

Para a construção da fronteira uniformizada de forma direta, em que as DMUs ineficientes formam um grupo de cooperação $W$, segundo o modelo DEAGSZ CCR orientado a insumos, tem-se a equação (2), conforme Gomes (2003), na qual $h_{R i} \mathrm{e} h_{i}$ são, respectivamente, as medidas de eficiência nos modelos DEAGSZ CCR e DEA CCR clássico; $W$ é o grupo de DMUs em cooperação; $r_{i j}=h_{i} / h_{j}$ é o fator de proporcionalidade resultante do emprego da estratégia proporcional. Para os modelos DEA CCR orientado a produtos e DEA BCC, em ambas as orientações, são derivadas expressões análogas (GOMES, 2003). 


$$
h_{R i}=h_{i}\left(1+\frac{\sum_{j \in W}\left[x_{j}\left(1-r_{i j} h_{R i}\right)\right]}{\sum_{j \notin W} x_{j}}\right)
$$

\section{MODELAGEM}

As unidades em avaliação são os centros de pesquisa da Embrapa, elegíveis para o recebimento de bolsas. Todos os 37 centros possuem pesquisadores líderes de projetos dos Macroprogramas (MP). Dessa forma, o modelo é composto por 37 DMUs.

As variáveis usadas foram os critérios selecionados pelo comitê da empresa para distribuir as bolsas. Como insumos foram selecionados quatro índices. O primeiro é um indicador ponderado que mede a complexidade dos projetos de pesquisa dos MP. Cada um dos MP possui características específicas quanto à estrutura de suas equipes e de seus arranjos institucionais e, dessa forma, complexidades diferentes. As notas usadas como ponderações na construção desse indicador de complexidade dos projetos foram aquelas eleitas pelo comitê interno, quais sejam, 10 para MP1, 5,38 para MP2, 0,77 para MP3 e 2,31 para MP6.

Como segundo insumo foi usado um indicador de produção científica da empresa. Esse indicador de produção é usado também nos modelos que avaliam o desempenho dos centros de pesquisa da Embrapa, como descrito em Souza et al. (1999). O índice agregado de produção é composto por 28 indicadores de produção, divididos em 4 categorias: produção científica; publicações técnicas; desenvolvimento de tecnologias, produtos e processos; difusão de tecnologias e imagem. Na agregação dessas categorias são usados os pesos definidos em reuniões gerenciais da empresa.

O terceiro insumo é o índice que mede a importância da participação de bolsistas nos projetos de pesquisa da unidade, conforme percepção em uma escala 0-100, caracterizada pela Superintendência de Pesquisa e
Desenvolvimento da Embrapa. Este critério foi levantado através de um questionário junto aos centros de pesquisa da empresa, e foi mensurado em função do portfolio de projetos de cada centro.

Finalmente, o quarto insumo é o índice que mede o potencial para supervisão dos bolsistas. Este é um critério mensurado em relação a cada projeto dos centros de pesquisa da Embrapa. Corresponde à soma dos doutores que estão disponíveis para fazer a supervisão de bolsistas PIBIC em todos os projetos. As observações foram normalizadas pela mediana da Embrapa.

A variável de produto do modelo DEA é o número de cotas de bolsas de cada centro de pesquisa. Até o final de 2005, o total de bolsas do PIBIC na Embrapa era de 303. Foram concedidas em 2006 mais 25 bolsas, perfazendo um total de 328.

O modelo DEA-GSZ foi rodado em duas etapas. $\mathrm{Na} 1^{\mathrm{a}}$ foram realocadas as 303 bolsas atuais. Nessa etapa, cada centro de pesquisa recebeu o mesmo número de cotas (distribuição eqüitativa, uniforme), ou seja, o output de cada DMU eqüivale a $303 / 37=8,1892$ cotas, que foram realocadas com o modelo DEA-GSZ. Como resultado, algumas unidades tiveram acréscimo, outras decréscimo e outras mantiveram a cota original.

$\mathrm{Na} 2^{\mathrm{a}}$ etapa foram alocadas as 25 novas bolsas concedias à empresa somente às unidades que tiveram redução de sua cota na etapa anterior. Foi igualmente usado o modelo DEA-GSZ para a alocação das 25 bolsas entre estas unidades. Este modelo foi constituído com um input adicional, qual seja, o número atual de cotas destes centros, de modo a minimizar o efeito de perda de cotas por parte das unidades. Assim como na etapa anterior, a cada uma destas unidades foi alocada uma distribuição uniforme de cotas, ou seja, o output de cada DMU eqüivale a $25 / \mathrm{n}^{\circ}$ de unidades com redução na $1^{\text {a }}$ etapa.

Foi selecionado o modelo DEA CCR, pois este é o modelo usado na avaliação de desempenho dos centros de pesquisa 
da empresa, com orientação a outputs, já que esta é a variável a realocar.

A motivação fundamental para o usos dos indicadores de liderança de projetos, importância da participação de bolsistas nos projetos, potencial de supervisão e produção científica como insumos é que essas variáveis servem como proxies de qualificação. Neste contexto, procura-se, intuivamente, contemplar com mais bolsas as unidades com mais potencial de pesquisa para atender à demanda do PIBIC. Isto obtém-se através de um programa voltado para maximização radial via outputs. DMUs que se mostram ineficientes em um modelo de programação com outputs uniformes representam unidades com potencial de pesquisa e orientação não contemplados totalmente com a distribuição uniforme das bolsas. A projeção na fronteira torna o sistema de distribuição eficiente, mas embute os problemas de sobras e faltas que devem ser resolvidos propriamente via métodos de otimização. Isto implementa-se pela redistribuição de sobras (folgas) a unidades ainda ineficientes depois do truncamento da solução DEA-GSZ, já que o objetivo é promover uma distribuição de cotas de bolsas que gere maior eficiência média global.

\section{RESULTADOS}

A Tabela 1 traz os dados usados e os resultados da abordagem aqui proposta. A coluna A representa o índice de produção científica; a coluna B o índice de potencial de supervisão dos bolsistas; a coluna $\mathrm{C}$ traz o valor do índice de importância dos bolsistas; a coluna D é o índice de complexidade dos projetos. $\mathrm{O}$ resultado do modelo DEA-GSZ é fracionário (coluna E) e número de bolsas é um valor inteiro. Para ficar coerente, os valores foram aproximados para o inteiro mais próximo. Caso a soma desses inteiros fosse inferior ao total, a diferença deveria ser alocada às Unidades de menor eficiência (1 cota a cada Unidade).

Ao calcular-se a diferença entre as cotas atuais e o resultado da etapa 1 verifica-se que 8 unidades tiveram redução de suas cotas (DMU_3, DMU_5, DMU_9, DMU_14, DMU_22, DMU_25, DMU_29, DMU_34). As 25 cotas atuais foram alocadas na $2^{\mathrm{a}}$ etapa a estas 8 unidades, de modo a minimizar o impacto da redução de cotas de bolsas.

A alocação final de bolsas às unidades é o somatório dos resultados das Etapas 1 e 2, conforme apresentado na Tabela 1, coluna $G$, na qual também encontra-se a diferença (coluna L) entre a distribuição atual (coluna I) e a nova distribuição das bolsas às unidades (coluna G), além das respectivas medidas de eficiência com base nessa alocação, calculadas com o software SIAD (Angulo Meza et al., 2005).

A distribuição de cotas proposta pela abordagem DEA-GSZ sempre produzirá uma eficiência média superior àquela com qualquer outra distribuição de cotas de bolsas, já que este é o objetivo do modelo: gerar uma situação de máxima eficiência. Isso vai ao encontro do objetivo da administração central de promover uso mais eficiente dos recursos disponíveis. Ressalta-se ainda que, caso a variável a realocar não fosse inteira, os resultados do modelo DEA-GSZ seriam os indicados, já que são os que produzem uma alocação mais justa, em que todas as DMUs são DEA eficientes. 
Tabela 1: Dados e resultados da abordagem proposta.

(continua)

\begin{tabular}{|c|c|c|c|c|c|c|c|c|c|c|c|}
\hline Unidade & A & B & C & D & $\mathbf{E}$ & $\mathbf{F}$ & G & $\mathbf{H}$ & I & $\mathbf{J}$ & $\mathbf{L}$ \\
\hline DMU_1 & 3,2440 & 14,2860 & 100,00 & 100,0000 & 22,9515 & 1 & 24 & 0,7186 & 13 & 0,1234 & 11 \\
\hline DMU_2 & 0,8490 & 7,1430 & 100,00 & 25,8913 & 8,8002 & 1 & 9 & 0,8115 & 5 & 0,1607 & 4 \\
\hline DMU_4 & 1,1816 & 100,0000 & 100,00 & 43,1552 & 13,0884 & 0,999998 & 14 & 0,7852 & 7 & 0,1478 & 7 \\
\hline DMU_5 & 1,1049 & 14,2860 & 100,00 & 30,2143 & 10,9602 & 0,999998 & 16 & 1,0000 & 24 & 0,6058 & -8 \\
\hline DMU_6 & 0,4168 & 14,2860 & 100,00 & 42,4347 & 6,4430 & 1 & 6 & 0,6687 & 4 & 0,2057 & 2 \\
\hline DMU_9 & 0,5551 & 10,7140 & 50,00 & 32,3664 & 6,7292 & 0,999999 & 9 & 0,9748 & 8 & 0,3090 & 1 \\
\hline DMU_10 & 1,9117 & 50,0000 & 100,00 & 54,6645 & 15,2159 & 0,999999 & 16 & 0,6968 & 9 & 0,1291 & 7 \\
\hline DMU_11 & 1,2262 & 14,2860 & 50,00 & 29,4938 & 7,9767 & 0,999997 & 8 & 0,6390 & 6 & 0,1424 & 2 \\
\hline DMU_12 & 0,1737 & 7,1430 & 100,00 & 5,0342 & 3,4359 & 1 & 3 & 0,9510 & 1 & 0,1555 & 2 \\
\hline DMU_13 & 1,0624 & 7,1430 & 50,00 & 35,9876 & 9,0848 & 1 & 9 & 0,6818 & 9 & 0,2242 & 0 \\
\hline DMU_14 & 1,0129 & 14,2860 & 25,00 & 17,9751 & 4,5392 & 0,999998 & 8 & 1,0000 & 32 & 1,0000 & -24 \\
\hline DMU_20 & 1,7567 & 7,1430 & 75,00 & 26,6305 & 8,9603 & 0,999999 & 9 & 0,7642 & 3 & 0,0944 & 6 \\
\hline DMU_21 & 0,9595 & 14,2860 & 75,00 & 15,8230 & 7,1162 & 1 & 7 & 0,7099 & 4 & 0,1420 & 3 \\
\hline DMU_22 & 0,3859 & 0,0000 & 0,00 & 20,1366 & 3,4359 & 1 & 5 & 1,0000 & 18 & 1,0000 & -13 \\
\hline DMU_23 & 0,8575 & 14,2860 & 37,50 & 46,0372 & 9,7128 & 1 & 10 & 0,7910 & 9 & 0,2250 & 1 \\
\hline DMU_24 & 1,6628 & 17,8570 & 75,00 & 29,4751 & 9,4456 & 1 & 9 & 0,6133 & 8 & 0,1729 & 1 \\
\hline DMU_25 & 0,2646 & 14,2860 & 87,50 & 12,2392 & 4,9567 & 1 & 7 & 1,0000 & 12 & 1,0000 & -5 \\
\hline
\end{tabular}


Tabela 1: Dados e resultados da abordagem proposta.

(conclusão)

\begin{tabular}{|c|c|c|c|c|c|c|c|c|c|c|c|}
\hline \multirow[b]{2}{*}{ Unidade } & A & $\mathbf{B}$ & $\mathbf{C}$ & D & $\mathbf{E}$ & $\mathbf{F}$ & $\mathbf{G}$ & $\mathbf{H}$ & I & $\mathbf{J}$ & $\mathbf{L}$ \\
\hline & $\begin{array}{c}\text { Prod. } \\
\text { científica }\end{array}$ & Superv. & Import. & Complex. & $\begin{array}{c}\text { Realocação } \\
\text { etapa } 1\end{array}$ & \begin{tabular}{|c|}
$\begin{array}{c}\text { Eficiência de } \\
\text { (E) }\end{array}$ \\
\end{tabular} & $\begin{array}{c}\text { Alocação final } \\
\text { etapas } 1 \text { e } 2\end{array}$ & $\begin{array}{c}\text { Eficiência } \\
\text { de (G) }\end{array}$ & $\begin{array}{c}\text { Alocação } \\
\text { atual }\end{array}$ & $\begin{array}{c}\text { Eficiência } \\
\text { de (I) }\end{array}$ & $\begin{array}{c}\text { Diferença } \\
(\mathrm{G})-(\mathrm{I})\end{array}$ \\
\hline DMU_26 & 0,6782 & 0,0000 & 75,00 & 1,4410 & 3,4359 & 1 & 3 & 1,0000 & 1 & 0,7763 & 2 \\
\hline DMU_27 & 1,1900 & 7,1430 & 100,00 & 1,4410 & 3,4359 & 1 & 3 & 1,0000 & 1 & 0,3898 & 2 \\
\hline DMU_28 & 0,4023 & 0,0000 & 37,50 & 7,1957 & 3,4359 & 1 & 3 & 1,0000 & 2 & 0,3109 & 1 \\
\hline DMU_29 & 0,5830 & 17,8570 & 50,00 & 5,0342 & 3,4359 & 1 & 5 & 1,0000 & 7 & 0,7811 & -2 \\
\hline DMU_30 & 0,7606 & 7,1430 & 100,00 & 11,5093 & 6,7067 & 1 & 7 & 0,9207 & 3 & 0,1643 & 4 \\
\hline DMU_31 & 0,7942 & 14,2860 & 75,00 & 17,9938 & 7,4495 & 0,999999 & 7 & 0,6733 & 3 & 0,1115 & 4 \\
\hline DMU_32 & 0,9390 & 7,1430 & 56,25 & 8,6367 & 4,7246 & 1 & 5 & 0,8461 & 4 & 0,2602 & 1 \\
\hline DMU_33 & 0,6970 & 21,4290 & 75,00 & 16,5435 & 7,1696 & 0,999999 & 7 & 0,7277 & 6 & 0,2497 & 1 \\
\hline DMU_34 & 1,5372 & 14,2860 & 100,00 & 25,9006 & 10,3078 & 1 & 15 & 1,0000 & 27 & 0,6908 & -12 \\
\hline DMU_35 & 0,8384 & 14,2860 & 75,00 & 25,1801 & 8,6321 & 1 & 9 & 0,7309 & 5 & 0,1605 & 4 \\
\hline DMU_36 & 0,1526 & 14,2860 & 37,50 & 7,9162 & 3,4359 & 1 & 3 & 0,8552 & 2 & 0,2813 & 1 \\
\hline DMU_37 & 1,2059 & 21,4290 & 75,00 & 12,9597 & 6,5869 & 0,999999 & 7 & 0,7564 & 5 & 0,2167 & 2 \\
\hline & & & & & $303 *$ & $1,0000 * *$ & $328 *$ & $0,8244 * *$ & $303 *$ & $0,3408 * *$ & \\
\hline
\end{tabular}

* Total; ** Média. 


\section{CONCLUSÕES}

O uso de modelos DEA-GSZ mostrouse bastante adequado a alocação de bolsas do PIBIC.

Os resultados do modelo proposto geraram uma eficiência média maior do que aquela obtida com os valores de cotas alocados pelo comitê interno da instituição. Isso é de especial interesse para a administração central da empresa, já que a alocação de cotas de bolsas do PIBIC aqui proposta permite o uso mais eficiente dos recursos, ou seja, permite identificar de forma ótima as unidades mais adaptadas para o processo de produção subjacente ao PIBIC.

\section{REFERÊNCIAS}

ANGULO MEZA, L.; BIONDI NETO, L.; SOARES DE MELLO, J.C.C.B.; GOMES, E.G. ISYDS - Integrated System for Decision Support (SIAD - Sistema Integrado de Apoio à Decisão): a software package for data envelopment analysis. Pesquisa Operacional, v. 25, n. 3, p. 493503, 2005.

BANKER, R.D.; CHARNES, A.; Cooper, W.W. Some models for estimating technical scale inefficiencies in data envelopment analysis. Management Science, v. 30, n. 9, p. 1078-1092, 1984.

BEASLEY, J.E. Allocating fixed costs and resources via data envelopment analysis. Technical Report, The Management School, Imperial College, London, 2001.

CHARNES, A.; COOPER, W.W.; RHODES, E. Measuring the efficiency of decision-making units. European Journal of Operational Research, v. 2, p. 429444, 1978.

COOPER, W.W.; SEIFORD, L.M.; TONE, K. Data Envelopment Analysis: a comprehensive text with models, applications, references and DEA-solver software. Boston: Kluwer Academic Publishers, 2000.
GOMES, E.G. Modelos de Análise de Envoltória de Dados com Ganhos de Soma Zero. 2003. Tese (Doutorado em Engenharia de Produção), COPPE/UFRJ, Rio de Janeiro, Brasil.

GOMES, E.G.; LINS, M.P.E. Modelling undesirable outputs with zero sum gains data envelopment analysis models. Journal of the Operational Research Society, 2007 (forthcoming).

GOMES, E.G.; SOARES DE MELLO, J.C.C.B.; LINS, M.P.E. Uniformização da fronteira eficiente em modelos de análise de envoltória de dados com ganhos de soma zero e retornos constantes de escala. Pesquisa Operacional, v. 25, n. 2, p. 261277, 2005.

KORHONEN, P.; SYRJÄNEN, M. Resource allocation based on efficiency analysis. Management Science, v. 50, n. 8, p. 1134-1144, 2004.

LINS, M.P.E.; GOMES, E.G.; SOARES DE MELLO, J.C.C.B.; SOARES DE MELLO, A.J.R. Olympic ranking based on a Zero Sum Gains DEA model. European Journal of Operational Research, v. 148, n. 2, p. 312-322, 2003.

LOZANO, S.N.; VILLA, G. Centralized resource allocation using data envelopment analysis. Journal of Productivity Analysis, v. 22, p. 143-161, 2004.

SOUZA, G.S.; ALVES, E.; ÁVILA, A.F.D. Technical efficiency in agricultural research. Scientometrics, v. 46, p. 141160, 1999.

YAN, H.; WEI, Q.; HAO, G. DEA models for resource reallocation and production input/output estimation. European Journal of Operational Research, v. 136, p. 19-31, 2002. 